1 Secretaria Municipal de Saúde de Fortaleza Fortaleza (CE), Brasil. killopes@gmail.com

2 Secretaria Municipal de Saúde de Fortaleza - Fortaleza (CE), Brasil. Universidade Estadual do Ceará (Uece) - Fortaleza (CE), Brasil.

lyvia_mesquita@hotmail. com

3 Universidade Estadual do Ceará (Uece) - Fortaleza (CE), Brasil.

andreacaprara1@gmail.com

4 Secretaria Municipal de Saúde de Fortaleza - Fortaleza (CE), Brasil. Universidade Estadual do Ceará (Uece) - Fortaleza (CE), Brasil.

bsbenevides@gmail.com

5 Secretaria Municipal de Saúde de Fortaleza - Fortaleza (CE), Brasil. Universidade Estadual do Ceará (Uece) - Fortaleza (CE), Brasil.

ronaldopg1@terra.com.br

\section{Organização do processo de trabalho no manejo da dengue em uma capital do Nordeste}

\author{
Organization of the work process in the handling of dengue in a \\ northeastern capital
}

Kilma Wanderley Lopes Gomes², Lyvia Patrícia Soares Mesquita², Andrea Caprara³, Bruno Souza Benevides4, Ronaldo Pinheiro Gonçalves 5

RESUMO Este estudo tem como objetivo descrever a organização do processo de trabalho na atenção primária como uma ferramenta de cuidado para os pacientes com suspeita de dengue. A situação de epidemias, em 2011 e 2012, impôs a execução do plano de contingência da dengue, elaborado de forma participativa com Equipes de Saúde da Família. As ações organizadas foram: qualificação dos profissionais, organização do serviço e monitoramento dos casos de dengue. A unidade atuou como a principal porta de entrada para a população. Foram notificados, respectivamente, 677 e 1081 casos em 2011 e 2012, sem o registro de óbitos. Implantar essas ferramentas no âmbito do serviço público constitui-se em um desafio.

PALAVRAS-CHAVE Serviços de saúde; Dengue; Atenção Primária à Saúde.

ABSTRACT This study aims to describe the organization of the work process in primary care as a care tool for patients with suspected dengue. The situation of epidemics in 2011 and 2012 imposed the execution of the dengue's contingency plan, elaborated in a participatory manner with Family Health Teams. The organized actions were: qualification of the professionals, service organization and monitoring of dengue cases. The unit served as the main gateway to the population. Were notified, respectively, 677 and 1081 cases in 2011 and 2012, without the register of deaths. Deploying these tools within the public service constitutes a challenge.

KEYWORD Health services; Dengue; Primary Health Care. 


\section{Introdução}

Inicia-se o ano e, concomitantemente, começam as chuvas e a dengue em todas as regiões do Brasil, demandando uma transformação do processo de trabalho, das rotinas dos serviços de saúde, do cotidiano das pessoas acometidas pela dengue e de suas famílias.

Mesmo sendo uma doença antiga, (re) emergente, ainda há limitações para reduzir tanto a taxa de infestação do seu vetor, o Aedes aegypti, como a morbimortalidade por dengue (TORRES, 2008). Tais questões instigam os gestores de saúde a buscarem soluções em vários aspectos da doença, no tocante à prevenção, ao tratamento e à cura.

Os óbitos por dengue, em sua grande maioria, são evitáveis, segundo consta nas Diretrizes Nacionais para a Prevenção e Controle de Epidemias de Dengue (DNPCD) (BRASIL, 2009B). Esse resultado, no entanto, está condicionado à qualidade da assistência prestada aos pacientes com suspeita de dengue e à organização da rede de serviços de saúde, refletindo como um forte indicador da qualidade da assistência.

A dengue acomete mais de 35 países e afeta populações de todas as idades e níveis socioeconômicos. Estima-se que 2,5 bilhões de pessoas vivam em países endêmicos em situação de risco. Em média, ocorrem entre 50 e 100 milhões de casos por ano, e a região das Américas não é uma exceção. A incidência de dengue aumentou 30 vezes nos últimos 50 anos (WHO, 2012, 2014).

Entre os anos de 2008 e 2012, mais de 1,2 milhão de casos foram notificados anualmente, incluindo 28.233 casos graves e 1.000 mortes (WHO, 2012, 2014).

No Ceará, onde a dengue é considerada uma doença endêmica desde 1986, ocorreu epidemias nos anos de 1987, 1994, 2001, 2008, 2011 e 2012. Fortaleza é a segunda cidade do Brasil que apresentou maior índice de notificações de casos e óbitos por dengue nos primeiros meses de 2012, ficando atrás apenas do Rio de Janeiro. Para que seja declarada situação de epidemia em um município, o Ministério da Saúde considera o índice mínimo de 300 casos para cada 100 mil habitantes. O município de Fortaleza atingiu 405,3 por 100 mil habitantes (O GLOBO, 2012).

Já em 2013, segundo o Informe SemanalDengue (1/11/2013), foram notificados 52.734 casos de dengue no Estado do Ceará, envolvendo todos os municípios (184). Fortaleza e Maracanaú foram os que mais registraram casos confirmados, com, respectivamente, $8.248(35,2 \%)$ e 1.639 (7,1\%) (FORTALEZA, 2013).

A complexidade desafiadora para o controle da dengue no mundo contemporâneo deve-se aos aspectos relacionados à permanência da dengue, tem influência multifatorial e, em sua maioria, fatores externos ao que convencionalmente é atribuído ao setor saúde (COELHO, 2008).

Nessa perspectiva, visualizam-se outros indicadores, para além do risco, que precisam ser incorporados a essa discussão: a questão das vulnerabilidades, que pode se expressar por uma dimensão individual, ou seja, pela qualidade e pelo aprendizado da informação que os indivíduos dispõem sobre seus problemas de saúde; a questão social, que revela como o coletivo se apropria das informações, do acesso aos meios de comunicação, dos recursos cognitivos e materiais disponíveis, e como participa das políticas de saúde; e a questão programática, que consiste na forma organizativa dos programas de responder ao controle das necessidades de saúde de forma efetiva (AYRES ET AL., 1999).

A Atenção Primária à Saúde (APS) é considerada a porta de entrada das pessoas no sistema de saúde, responsável pela coordenação do cuidado e pela realização do primeiro atendimento da maioria dos casos de dengue clássica, assim como o seguimento diário e domiciliar dos pacientes junto a Equipes de Saúde da Família (EqSF) (ANDRADE, 2004).

Deve haver disponibilidade de recursos, garantia de estabelecimentos de saúde que ofereçam serviços de promoção, 
prevenção, diagnóstico, tratamento, gestão de casos, reabilitação e cuidados paliativos de acordo com as necessidades de saúde. Dessa forma, pode-se repensar em um modelo de atenção à saúde efetivo e capaz de acompanhar longitudinalmente sua população em qualquer problema de saúde, seja crônico ou agudo (IBGE, 2010).

O acesso ofertado nas unidades de Atenção Primária à Saúde (APS) pode garantir tanto o primeiro atendimento como o monitoramento das consultas subsequentes aos pacientes com suspeita de dengue. Para que isso ocorra, é necessário que o planejamento da gestão central esteja conectado ao local, com o propósito de organizar o fluxo da assistência, a logística de apoio e o processo de trabalho das equipes de saúde, levando em conta as necessidades da população.

Toma-se como pressuposto que a procura da cura do paciente com dengue é um percurso que vai além da escolha individual, envolve o coletivo, uma rede social de apoio e a forma como o sistema de saúde se organiza para promover o acesso à população. Desse modo, este estudo, na perspectiva de contribuir com aspectos relevantes da prática clínica e da organização do serviço para atender às demandas do paciente com dengue, tem como objetivo descrever a organização do processo de trabalho na atenção primária como uma ferramenta de cuidado aos pacientes com suspeita de dengue.

\section{Relato de experiência}

A experiência a ser relatada ocorreu em uma unidade de APS da cidade de Fortaleza/ Ceará. Uma cidade heterogênea, com grandes desigualdades sociais, em que cerca de um terço da população mora em favelas (IBGE, 2010). Apresenta um Índice de Desenvolvimento Humano (IDH) de 0,754.

Os serviços de saúde estão organizados e distribuídos em sete Secretarias Regionais (SR): Centro, I, II, III, IV, V, VI
(FORTALEZA, 2014). A SR V fica localizada na região Sudoeste da cidade e apresenta uma população com um dos mais baixos IDH de Fortaleza $(0,44)$. É uma das áreas que mais apresenta casos de dengue. A experiência a ser relatada ocorreu em uma unidade de APS dessa SR.

Em 2011 e 2012, a cidade de Fortaleza vivenciou epidemias de dengue. Essa situação impôs a execução do plano de contingência da dengue, organizado pela gestão central e compartilhado com gestores e profissionais que atuam nos serviços de saúde.

O plano foi elaborado com base nos eixos estratégicos das Diretrizes Nacionais para a Prevenção e Controle de Epidemia de Dengue (DNPCD). No tocante à assistência ao paciente, algumas diretrizes foram elencadas para garantir a assistência adequada e oportuna, buscando como foco prioritário reduzir a letalidade das formas graves da dengue. Entre as ações implantadas, destacam-se: a classificação de risco para prioridade de atendimento, a organização dos serviços de saúde e as estratégias para enfrentamento de uma epidemia de dengue.

Para execução do plano, a gestão central articulou, junto aos gestores de unidades de APS e hospitais municipais, reuniões para sensibilizar sobre a problemática da epidemia de dengue no município e sobre estratégias a serem implementadas a partir do plano de contingência.

O foco deste estudo é descrever como ocorreu a organização do processo de trabalho em uma unidade de APS, tendo como premissa que essa é uma ferramenta de cuidado fundamental para o manejo adequado aos pacientes com suspeita de dengue.

\section{Caracterizando a unidade de saúde}

A unidade é composta por 114 profissionais, cinco EqSF, sendo três completas, 44 microáreas, sendo $54 \%$ de cobertura da Estratégia Saúde da Família para seu território de abrangência sanitária. 
O plano local da unidade foi elaborado de forma participativa, em meados de fevereiro de 2011, a partir das orientações da gestão. Inicialmente, a coordenação da unidade de saúde reuniu seus profissionais para discutir sobre a proposta municipal para o controle da dengue, havendo o envolvimento de todos no processo. Ocorreu sensibilização desses profissionais para alerta da dengue no território e de uma possível epidemia no município, além de reflexão sobre o papel a ser desenvolvido por cada trabalhador e apresentação dos indicadores de incidência e letalidade, com enfoque para o território da unidade. As ações organizadas para a unidade foram: 'Qualificação dos profissionais sobre manejo clínico' e 'Organização do serviço para atender dengue e monitoramento dos casos de dengue'.

\section{Qualificação dos profissionais so- bre manejo clínico para médicos e enfermeiros}

Um profissional da unidade foi qualificado na proposta do Ministério da Saúde 'Dengue em 15 Minutos', e atuou como facilitador do processo de multiplicação em nível local com todos os profissionais médicos e enfermeiros que iriam participar do atendimento, com classificação de risco da unidade. $O$ treinamento foi iniciado com aplicação de um pré-teste para avaliar como o assunto dengue estava presente no cotidiano. Após a discussão dos resultados, todo o plano que envolve o manual de controle da dengue, produzido pelo Ministério da Saúde, foi repassado, elaborado em conjunto um plano de ação local, discutido o fluxograma com classificação de risco e o comprometimento de capacitar os outros integrantes da equipe.

\section{Capacitação de técnicos e auxiliares de enfermagem}

Todo o corpo de enfermagem e outros profissionais de nível médio (recepcionistas, porteiros, atendentes) foram qualificados para identificação de possíveis pacientes com suspeita de dengue, de forma a não voltar nenhum paciente, seja da área de abrangência ou fora da área de atendimento de acolhimento, como fila de farmácia ou de marcação de exames, ou mesmo na espera de consultas programadas. A equipe de enfermagem também foi sensibilizada quanto ao atendimento dos sinais vitais, sinais de alarme e hidratação (oral e venosa), oferecendo o exame da prova do laço na sala de triagem, antes mesmo da entrada do paciente no consultório. A equipe foi orientada sobre a manutenção diária do espaço reservado para hidratação oral, bem como a oferecer soro oral aos pacientes e a monitorar a aceitação.

\section{Capacitação de Agentes Comunitá- rios de Saúde (ACS)}

Foi elaborado um instrumento para identificação de casos da doença na área de abrangência das equipes, segundo o qual o ACS, ao identificar alguma sintomatologia de possível quadro de dengue, encaminharia imediatamente o paciente à sala de triagem. E para monitoramento nos domicílios dos pacientes em acompanhamento domiciliar pelos ACS, conforme recomenda o protocolo do Ministério da Saúde.

Foram estabelecidas parcerias com a comunidade visando à implantação de ações como: a campanha do quintal limpo pelos ACS e agentes sanitaristas nas áreas de maior índice de positividade e de alerta da dengue nos domicílios; ações de educação em saúde; e o monitoramento dos casos nas áreas de abrangência da unidade de saúde pela vigilância epidemiológica. Houve palestras junto às escolas e foi divulgado pelos ACS o fluxo de horário e atendimento da unidade para atenção aos casos suspeitos de dengue.

\section{Organização do serviço para atender dengue}

Para a consecução dos objetivos, foram elaborados: um prontuário de atendimento 
diário específico para os casos suspeitos de dengue; utilização de um receituário padronizado com informes sobre sinais de alarme e distribuição do soro de reidratação oral e outros líquidos por volume e horário; implementação da utilização na rotina do cartão de acompanhamento diário. As equipes se organizaram para realizar o acolhimento por classificação de risco diariamente. Cada equipe ficou responsável pelo atendimento dos casos da doença em um dia fixo da semana, visando garantir o acesso (tanto à primeira consulta quanto à consulta de retorno), o acompanhamento e a vinculação dos pacientes às equipes. Com isso, conseguiu-se manter e acompanhar os pacientes classificados no grupo A e B de dengue e encaminhar os pacientes com sinais de alarme para outras unidades hospitalares de referência.
Foi ampliada a sala de observação e hidratação, aumentando o número de leitos para observação na própria unidade.

$\mathrm{Na}$ área logística, o apoio de transporte foi ampliado e assegurado o estoque de insumos como soro de reidratação oral, entre outros. Além disso, ficou acordado com o laboratório responsável pelo posto de coleta local a melhoria da coleta de exames com ampliação do horário de atendimento e agilidade na entrega de resultados no dia, itens fundamentais para um adequado atendimento ao paciente.

\section{Resultado e discussão}

Com relação aos casos de dengue atendidos na unidade, foram notificados, respectivamente, 677 e 1081 casos em 2011 e 2012, sem o registro de óbitos nesse período.

Tabela 1. Número de casos notificados com suspeita de dengue, nos anos de 2011 e 2012, na unidade de Atenção Primária à Saúde

\begin{tabular}{|c|c|c|c|c|c|c|c|c|c|c|c|c|c|}
\hline & JAN & FEV & MAR & ABR & MAI & JUN & JUL & AGO & SET & OUT & NOV & DEZ & TOTAL \\
\hline 2011 & 13 & 56 & 165 & 251 & 133 & 31 & 5 & 8 & 9 & 1 & 0 & 5 & 677 \\
\hline 2012 & 0 & 7 & 2 & 133 & 610 & 239 & 21 & 2 & 4 & 0 & 0 & 0 & 1018 \\
\hline
\end{tabular}

Fonte: Fortaleza, 2013

Devido ao grande número de casos notificados, foi elaborada uma planilha de investigação diária compactada, na qual os profissionais preenchiam os dados fundamentais dos pacientes e, ao fim de cada turno de trabalho, eram recolhidas das salas de acolhimento e repassadas para a ficha de notificação e para livro de controle epidemiológico, dando agilidade ao atendimento do profissional e facilitando a obtenção dos dados diários.

\section{Atenção primária como porta de entrada}

Partindo do princípio de que a APS é a principal porta de entrada ao sistema de saúde, ela se apresenta como papel de filtro para o acesso a outros serviços, assumindo a responsabilidade longitudinal pelo paciente ao longo da vida, independentemente da ausência ou presença de doença (STARFIELD, 2002). A unidade atuou como a principal porta de entrada para a população de sua responsabilidade sanitária, além de realizar atendimentos para outras unidades de atenção primária.

A garantia de cuidado integral a partir da estruturação dos âmbitos físicos, psíquicos e sociais da saúde, dentro dos limites de atuação profissional, é fundamental para a resolutividade dos problemas da população. Na experiência vivida, procurou-se, a partir 
da organização e do envolvimento de todos os trabalhadores, a realização do monitoramento do paciente em todos os espaços de cuidado, fosse ele unidade de saúde ou domiciliar, garantindo, assim, não somente $o$ acesso, mas a integralidade do cuidado.

Um sistema de saúde organizado, que prioriza a APS como coordenadora do cuidado, propõe a estruturação dos serviços de saúde com o intuito de integrar todas as intervenções de promoção, prevenção, reabilitação e cura, a partir das necessidades da população. O Brasil vem, por meio da Atenção Básica, qualificando o sistema de saúde com foco na equidade social, com corresponsabilidade entre a população e o setor público, de forma a investir na Estratégia Saúde da Família (ESF) (ANDRADE, 2004).

Segundo Giovanella e Mendonça (2012), a APS é reconhecida mundialmente como a base para um novo modelo assistencial de sistemas de saúde. Contudo, há diferença de recursos e abordagens. Os países europeus preconizam a atenção primária como o serviço de primeiro contato, integrado a um sistema de saúde de acesso universal. Já os países em desenvolvimento ainda fazem uma atenção primária com recursos de baixa densidade tecnológica e sem integrar de fato aos outros níveis de atenção secundário e terciário.

O Ministério da Saúde tem como premissa a Atenção Básica para dar soluções efetivas às necessidades da população:

Um conjunto de ações de saúde, no âmbito individual e coletivo, que abrange a promoção e a proteção da saúde, a prevenção de agravos, o diagnóstico, o tratamento, a reabilitação, redução de danos e a manutenção da saúde com o objetivo de desenvolver uma atenção integral que impacte na situação de saúde e autonomia das pessoas e nos determinantes e condicionantes de saúde das coletividades. (BRASIL, 2011).

A reorganização da Atenção Básica, por meio da ESF, é guiada pelos princípios organizativos do SUS, como estratégia para expansão e qualificação do sistema de saúde. Nesta perspectiva, faz-se necessário no cenário da dengue ampliar a resolutividade a partir do envolvimento das pessoas, participando e se informando de forma efetiva (BRASIL, 2011).

Entre as suas finalidades, destaca-se aquela que tem por objetivo resolver os problemas de saúde das populações de maior frequência e relevância. É o contato preferencial dos usuários com o sistema de saúde. Orienta-se pelos princípios da universalidade, acessibilidade (ao sistema), continuidade, integralidade, responsabilização, humanização, vínculo, equidade e participação social.

Partindo do princípio de que a dengue é uma doença aguda e endêmica em muitas regiões, inclusive no nosso município, fazem-se necessários: a realização do diagnóstico, o tratamento e o manejo de início precoce em todos os níveis de atenção, bem como o estado de 'alerta' para os sinais de alarme, tanto pelos profissionais como pela população, ações essas consideradas fundamentais para o sucesso de um caso de dengue e, consequentemente, para a redução dos casos graves que evoluem para o óbito (BRASIL, 2012).

Outro aspecto suscitado foi a necessidade de mostrar e valorizar como se dá a construção dos processos entre e com as equipes na perspectiva de organização do serviço, para atender e receber o paciente com suspeita de dengue, enfocando que, além da organização do processo de trabalho, a pactuação para realizar a vigilância sistemática dos pacientes com dengue atendidos na unidade, a garantia e a otimização dos recursos disponíveis são essenciais para a qualidade da assistência.

No início, houve resistência dos profissionais em modificar a agenda, como também a presença de uma demanda elevada, em virtude de o serviço ficar como referência para outras unidades de atenção primária. Situações-limite semelhantes foram encontradas por Silva (2013), ao compreender a percepção dos profissionais implicados na organização do serviço.

Implantar essas ferramentas no âmbito do serviço público constitui-se em um grande 
desafio para os gestores de saúde, pois a dengue é mais um entre muitos outros problemas sobre os quais se há de agir. O componente da assistência na saúde que consta nas DNPCD (BRASIL, 2009A) propõe focar a atenção na estrutura e na organização do serviço e na qualificação profissional, mas não põe em perspectiva aspectos inerentes à pessoa doente, que influenciam a tomada de decisão e suas escolhas terapêuticas. Na organização do processo de trabalho em saúde, têm-se ressaltado os espaços de produção de bens e serviços para os usuários, assim como espaços de valorização do potencial de cada profissional envolvido. Situações adversas são vivenciadas no dia a dia, e o que se planeja não corresponde exatamente ao que se faz, porque há outras implicações que dependem da subjetividade, da singularidade de cada pessoa, no que tange ao papel do paciente ou do profissional, do coletivo, dos acordos e pactos realizados entre os profissionais e a comunidade (BRASIL, 2009A).

Merhy et al. (1997) referem que o foco essencial do trabalho em saúde seriam as tecnologias leves, entendidas como parte destas a comunicação de forma singular entre profissionais e usuários, favorecendo o vínculo, a escuta, a informação e a relação. Contudo, essa tecnologia não tem sido fácil de ser implantada pelos sistemas de gerenciamento dos serviços de saúde, nem pelos profissionais junto às pessoas que procuram atenção. Um dos fatores que podem estar contribuindo são os protocolos e modelos de atenção fechados, muitas vezes verticalizados, que não valorizam tal tecnologia, nem mesmo pelos gestores na hora de planejar.

A pactuação realizada entre os trabalhadores para o plano de controle da unidade foi construída entre várias idas e vindas para trabalhar as agendas, negociar as trocas e ausências entre os profissionais de saúde, preenchimento correto do cartão e prontuário. Aspectos fundamentais para a realização dos encontros foram os diálogos e vínculos estabelecidos entre todos, tipo parceria.
Silva (2012), ao realizar um estudo com cartografia em uma unidade de atenção primária, observou que o encontro entre os profissionais favorece o agenciamento da mudança de uma ação somente assistencialista para uma prática direcionada ao cuidado.

Oliveira e Casanova (2009) argumentam a importância da vigilância à saúde na reorientação da atenção básica, levando em conta a territorialização, uma ferramenta para conhecer o território de atuação não somente espacial, mas com suas peculiaridades, subsídio para conhecer como as pessoas vivem, trabalham, adoecem, como se organizam, como é a real condição de vida, determinantes que as fazem adoecer e promover saúde, o que pensam sobre a saúde e, enfim, a importância da atuação da EqSF no mapeamento e apropriação do contexto real do território dinâmico.

Durante a organização do serviço da unidade de saúde, não bastou somente reunir as equipes, mas envolvê-las e fazê-las corresponsáveis pelo processo.

Sales (2008) defende o fortalecimento da relação entre profissionais e usuários, constatada em seu trabalho em uma cidade do Ceará como uma deficiência para motivar a corresponsabilidade do paciente na construção de estratégias de controle da dengue.

O uso e a resposta dos serviços de saúde podem ser indicadores de acesso, mas por si só não bastam para explicar a qualidade da atenção, que resulta de uma multiplicidade de fatores, sejam no campo comportamental das pessoas que procuram o serviço para receber cuidado, na organização e na estrutura do serviço ou na qualificação do profissional que o conduz dentro do sistema de saúde (TRAVASSOS; MARTINS, 2004).

\section{Considerações}

Diante da experiência relatada, consideramos que a relação e a inter-relação no cotidiano das equipes são aspectos importantes que devem ser incorporados nas estratégias de organização 
do processo de trabalho. São motivadores para o envolvimento de todos os atores, principalmente para o enfrentamento de eventos inesperados ou epidêmicos, como é o caso da dengue, que demanda compromisso, reorganização da prática e participação efetiva. Outro ponto importante é o investimento nas condições estruturais. Não há possibilidade de execução de um plano de controle da dengue sem apoio da logística, incluindo a capacitação profissional.

O conhecimento da situação da doença em cada comunidade, o monitoramento dos casos e a realização da vigilância à saúde pelos agentes comunitários e de endemias se fazem necessários para incrementar a efetividade do plano local de controle da dengue.

A articulação entre a vigilância epidemiológica e as equipes que prestam atenção à saúde é fundamental para o cumprimento dos indicadores diários, de forma que estejam sempre em posição de 'alerta', facilitando tomadas de decisão em tempo hábil.

É possível prevenir e atuar com êxito diante da dengue, implementando indicadores para o manejo da doença que possam ser discutidos junto às equipes no dia a dia, de forma a qualificar o acesso do paciente e seu seguimento pelas EqSF.

\section{Referências}

ANDRADE, L. O. M.; BARRETO, I. C. H. C.; FONSECA, C. D. A estratégia saúde da família. In: DUNCAN, B. B.; SCHMIDT, I. M.; GIUGLIANI, E. R. J. (Org.). Medicina ambulatorial: condutas na atenção primária baseada em evidência. Rio de Janeiro: Artmed, 2004.

AYRES, J. R. C. M. et al. Vulnerabilidade e prevenção em tempos de Aids. In: BARBOSA, R.; PARKER, R. (Org.). Sexualidade pelo avesso: direitos, identidades e poder. Rio de Janeiro: Relume Dumará, 1999, p. 50-71.

Ministério da Saúde. Grupo de trabalho de Gestão da Câmara Técnica da Comissão Intergestores Tripartite. Conselho Nacional de Secretarias Municipais de Saúde. Conselho Nacional de Secretários de Saúde. Anexo - Diretrizes para Organização da Rede de Atenção à Saúde do SUS. Brasília, DF, 2010. Disponível em: <http://bvsms.saude.gov.br/bvs/ saudelegis/gm/2010/anexos/anexos_prt4279_30_12_2010. pdf > . Acesso em: 30 nov. 2013.

Ministério da Saúde. Portaria n ${ }^{\circ} 2.488$, de 21 de outubro de 2011. Aprova a Política Nacional de Atenção Básica, estabelecendo a revisão de diretrizes e normas para a organização da Atenção Básica, para a Estratégia Saúde da Família (ESF) e o Programa de Agentes Comunitários de Saúde (PACS). Diário Oficial [da] União. Brasília, DF, 2011. Disponível em: <http://bvsms.saude.gov.br/bvs/saudelegis/gm/2011/ prt2488_21_10_2011.html>. Acesso em: 15 fev. 2013.
Ministério da Saúde. Secretaria de Atenção à Saúde, Diretoria Técnica de Gestão. Diretrizes para a organização dos serviços de atenção à saúde em situação de aumento de casos ou de epidemia de dengue. Brasília, DF, 2012.

Ministério da Saúde. Secretaria de Atenção à Saúde. Política Nacional de Humanização da Atenção e Gestão do SUS: trabalho e redes de saúde. Brasília, DF: Ministério da Saúde, 2009a.

Ministério da Saúde. Secretaria de Vigilância em Saúde. Departamento de Vigilância Epidemiológica. Diretrizes nacionais para prevenção e controle de epidemias de dengue. Brasília, DF, 2009b.

COELHO, G. E. Dengue: desafios atuais. Epidemiol. Serv. Saúde, Brasília, DF, v. 17, n. 3, set. 2008.

FORTALEZA. Informe Semanal Dengue - 2013, 29 nov. 2013. Disponível em: <http://sala situacao.saude.ce.gov. br/index.php/publicacoes/nota-tecnica>. Acesso em: 5 nov. 2013. (Nota Técnica).

FORTALEZA. Prefeitura Municipal. Regionais. Disponível em: <http://www.fortaleza.ce. gov.br/regionais >. Acesso em: 10 fev. 2015 .

FORTALEZA. Prefeitura Municipal. SIMDA - Sistema de Monitoramento Diário de Agravos. Disponível em: < 
http://tcl.sms.fortaleza.ce.gov.br/simda/dengue/unidadeTipo>. Acesso em: 20 jan. 2014.

GIOVANELLA, L. MENDONÇA, M. H. M. Atenção Primária à Saúde. In: GIOVANELLA, L.; ESCOREL, S.; LOBATO, L. V. C. et al. (Org.). Políticas e Sistema dede Saúde no Brasil. 2 ed. Rio de Janeiro: Editora FIOCRUZ, 2012.

\section{INSTITUTO BRASILEIRO DE GEOGRAFIA E} ESTATÍSTICA (IBGE). Contagem da população 2010. Rio de Janeiro: IBGE, 2010. Disponível em: <http://cidades.ibge.gov.br/xtras/uf.php? coduf $=23>$. Acesso em: 30 nov. 2013.

MERHY, E. E.et al. Em busca de ferramentas analisadoras das tecnologias em saúde: a informação e o dia a dia de um serviço, interrogando e gerindo trabalho em saúde. In: MERHY, E. E.; ONOCKO, R. (Org.). Praxis en salud: un desafío para lo público. São Paulo: Hucitec, 1997. p. 113-149.

O GLOBO. Fortaleza tem o $2^{\circ}$ maior número de notificações de dengue do Brasil. Disponível em: <http:// gl.globo.com/ceara/noticia/2012/05/fortaleza-tem-o2-maior-numero-de-notificacoes-de-dengue-do-brasil html>. Acesso em: 22 ago. 2012.

OLIVEIRA, C. M.; CASANOVA, A. O. Vigilância da saúde no espaço de práticas da atenção básica. Ciênc. saúde colet., Rio de Janeiro, v. 14, n. 3, maio/jun. 2009, p. $929-936$

PAIM, J. et al. sistema de saúde brasileiro: história, avanços e desafios. In: PAIM, J. (Org.) Saúde no Brasil. Salvador: Instituto de Saúde Coletiva, Universidade Federal da Bahia, 2011. p. 11-31.

SALES, F. M. S. Ações de Educação em Saúde do Pará. Prevenção e Controle da dengue: um Estudo em Icaraí, Caucaia, Ceará. Ciênc. saúde colet., Rio de Janeiro, v. 13, n. 1, fev. 2008. Disponível em: <http://www.scielo.br/ scielo.php> Acesso em: 7 mar. 2015.

SILVA, G. A. P., SILVA, L. M. V. Organização das Práticas de Vigilância em Saúde em um Sistema Local. Revista Baiana de Saúde Pública. Salvador, v. 37, n. 1, p. 57-73, jan./mar. 2013. Disponível em: < http://files. bvs.br/upload/S/0100-0233/2013/v37nl/a3820.pdf>. Acesso em: 9 mar. 2015

SILVA, M. R. F. Linhas de cristalização e de fuga nas trilhas da Estratégia de Saúde da Família. Uma cartografia da micropolítica. 2012. 199 f. Tese (doutorado em Saúde Coletiva) - Universidade Federal do Ceará, Fortaleza. 2012.

STARFIELD, B. Atenção primária: equilíbrio entre necessidades de saúde, serviços e tecnologia. Brasília, DF: Organização das Nações Unidas para a Educação, a Ciência e a Cultura: Ministério da Saúde, 2002.

TEIXEIRA, M. G.; BARRETO, M. L.; GUERRA, Z. Epidemiologia e medidas de prevenção do dengue. Informe epidemiológico do SUS, Salvador, v. 8, n. 4, 1999, p. 5-33.

TORRES, E. M. Dengue. Estudos Avançados, São Paulo, v. 22 , n. 64 , dez. 2008 , p. $33-52$

TRAVASSOS, C.; MARTINS, M. Uma revisão sobre os conceitos de acesso e utilização de serviços de saúde. Cad. Saúde Pública, Rio de Janeiro, v. 20, supl. 2, 2004, p. 190-198.

WORLD HEALTH ORGANIZATION (WHO). Global strategy for dengue prevention and control 2012. Geneva, 2012. Disponível em: <http://apps.who.int/iris/bitstream/10665/75303/1/9789241504034_eng.pdf >. Acesso em: 15 maio 2013

State of the art in the Prevention and Control of Dengue in the Americas. Disponível em: < www.paho. org/hq/index.php.>. Acesso em: 20 jan. 2015.

Recebido para publicação em setembro de 2014

Versão final em março de 2015

Conflito de interesse: inexistente

Suporte financeiro: não houve 